\title{
Breast Surgery Fellowship: Need of Contemporary Times
}

\author{
Rufina Soomro \\ Department of General and Breast Surgery, Liaquat National Hospital \& Medical College, Karachi, Pakistan
}

Breast cancer is the most common cancer in women worldwide. Age standardised incidence of breast cancer is $43.9 / 100,000$ worldwide with over 2 million new cases diagnosed in 2018. It has a mortality of $23.2 / 100,000$. In Pakistan, a country with a population of over 200 million, the total new cancer cases diagnosed in 2018 were 173,937 ; and breast cancer accounts for 34,066 $(19.6 \%)$. Among this, 92,693 were females and breast cancer is the most common cancer diagnosed in females $(36.08 \%)$. The 5 -year prevalence of breast cancer is 73,046. In 2018 alone, overall cancer-related deaths reported in Pakistan are 118,442. Breast cancer share is $17,158(14.5 \%)$, which is the number one cause of cancer-related mortality (2018). ${ }^{1}$

The rising incidence of breast cancer in Pakistan is alarming. Unfortunately, breast cancer is diagnosed at a younger age and at an advanced stage in Pakistan. ${ }^{2}$ Generally, an earlier diagnosis ensures optimal treatment and maximal survival benefit. There are various reasons for delayed presentation; namely low literacy rate, lack of awareness, psycho-social taboos, beliefs in alternate therapies, and lack of diagnostic and treatment facilities. The most significant factor is the shortage of adequately trained manpower to deal with the rising burden of this disease.

To address the enormous burden of preventable mortality caused by breast cancer, we need to tackle it both nationally and at a community level by increasing awareness, in which the medical community and media have to play a major role. "National Breast Cancer Screening Program" is a dream-project. Adequate awareness and training at the basic healthcare level is important for timely referral to a specialist. Above all, we need to have appropriate facilities for early diagnosis and adequate treatment of these women.

With $49.2 \%$ of female population in Pakistan ${ }^{3}$, there are not enough breast cancer centres, in both the private and public sectors. For most of those which do exist, the quality of service is not at par with international standards. CPSP, being the only premier postgraduate centre at a national level, realised the "need for change"

Correspondence to: Dr. Rufina Soomro, Department of General and Breast Surgery, Liaquat National Hospital \& Medical College, Stadium Road, Karachi, Pakistan

E-mail: rufina.soomro@hotmail.com

Received: September 02, 2019; Revised: October 16, 2019;

Accepted: October 16, 2019 and decided to get people trained in the management of breast cancer.

Comprehensive breast cancer management is provided through multidisciplinary teams comprising of a list of specialties, on top being surgeons, radiologists, pathologists and oncologists. Fellowships have already been started in diagnostics and oncology. But, we do not have adequately trained surgeons to do diagnostic workup and surgeries as done worldwide. Here, because of a lack of adequately trained breast surgeons, the general surgeons, without specialised training in breast surgery, are still offering suboptimal treatment which has no place in the modern evidence-based practice of breast cancer. Such practices translate into significant physical and psychological morbidity, which can be avoided.

In Pakistan, trained breast surgeons are need of the time to meet the challenge of breast cancer management. Making a curriculum in such a specialised subject, getting it approved and implemented, is a challenge, which CPSP accepted; and introduced a $2^{\text {nd }}$ FCPS in breast surgery.

This fellowship will empower the already trained surgeons to acquire necessary knowledge and skills to manage patients in a multi-disciplinary forum. MDT (multi-disciplinary team) has been shown to significantly influence clinical decision-making and treatment recommendations. ${ }^{4}$ In Pakistan, where there are no mass screening services available, breast surgeons not only help in the diagnosis of breast cancer, but play a pivotal role in the complete sequence of management of patients.

The designed curriculum is integrated and multidisciplinary in line with modern teaching and training. The fellowship in breast surgery will help the trainees to learn less invasive diagnostic methods of palpable and impalpable lesions. They will be trained to offer the right surgery for the right patient to have minimum morbidity; and will hopefully improve the aesthetic and oncological outcome of the patients. The Fellow is required to have knowledge and skills about all related services including radiology, pathology, oncology and plastic surgery. Multidisciplinary meetings are mandatory component of the training centres; and their Fellows are expected to be well-versed with research, data keeping, and raising awareness. Assessment of Fellows is based on both standard CPSP examination system and workplace. This will bring about a trained breast surgeon parallel to getting training abroad. 
This fellowship will serve as a catalyst for many to improve their services so they also acquire necessary skills and resources for training. The Fellows trained from this programme will spread out to various hospitals all across Pakistan. The persona of surgeons and adequate training of these new stars will help in the establishment of proper breast care services in whichever city they practice. With time, the first and second generations of trained surgeons are going to grow and will result in improve breast care services all over Pakistan. Such institutes with trained personnel can have a national level reputation inspiring others to join the noble cause.

For the sustainability of this programme, CPSP must not compromise on quality in recognition of training centres and trainers. The emphasis on improving facilities and trained personnel is of paramount importance; and this in turn, will improve patient care and only then, we will be able to improve survival.

Sustainability of fellowship is assured if you have developed an internal pipeline system which have both under- and post-graduate education. It can only thrive, if it recruits committed Fellows who are willing to be a part of highly skilled and high-demand field. ${ }^{5}$ These Fellows serve as a catalyst in transforming the general cadre of discipline into a highly specialised field. Thus, it is the duty of the supervisor to fine-tune these programmes in accordance with individual needs. The curriculum needs to be dynamic and should be able to cater the needs of the individual trainees. Feedback provided by the Fellows about the programme needs to be timely incorporated in the subsequent revisions.

With increasing numbers of trained people, one would be able to develop research-based national guidelines according to our needs, which would account for challenges that we face due to different tumor biology and financial constraints of our population. Through scientific research, understanding, and good data keeping, we can aim at collaboration with international organisations to bring funding through research in Pakistan.

We hope and pray that our efforts in the future will help us to achieve our goal to provide best healthcare service to the people of our community.

\section{REFERENCES}

1. The Global Cancer Observatory. May 2019. International Agency for research on cancer. WHO. https://gco.iarc.fr/today/ data/factsheets/populations/586-pakistan-fact-sheets.pdf

2. Soomro R, Faridi S, Khurshaidi N, Zahid N, Mamshad I. Age and stage of breast cancer in Pakistan: An experience at a tertiary care center. J Pak Med Assoc 2018; 68:1682-5.

3. Live Pakistan population (2019). Country meters. https:// countrymeters.info/en/Pakistan

4. Croke JM, El-Sayed S. Multidisciplinary management of cancer patients: Chasing a shadow or real value? An overview of the literature. Curr Oncol 2012; 19:e232-8.

5. Herr KD, Hanna TN, Khurana B, Johnson JO, Sodickson AD. Creating and sustaining a successful fellowship program: Challenges and solutions. Curr Probl Diagn Radiol 2017; 46: 95-9. 